\title{
Multiplatform Instantaneous GNSS Ambiguity Resolution for Triple- and Quadruple-Antenna Configurations with Constraints
}

\author{
Peter J. Buist, ${ }^{1}$ Peter J. G. Teunissen, ${ }^{1,2}$ Gabriele Giorgi, ${ }^{1}$ and Sandra Verhagen ${ }^{1}$ \\ ${ }^{1}$ DEOS, Delft University of Technology, Kluyverweg 1, 2629 HS Delft, The Netherlands \\ ${ }^{2}$ Department of Spatial Sciences, Curtin University of Technology, Perth WA 6845, Australia \\ Correspondence should be addressed to Peter J. Buist, p.j.buist@tudelft.nl
}

Received 27 February 2009; Revised 15 June 2009; Accepted 5 August 2009

Recommended by J. Riba

Traditionally the relative positioning and attitude determination problem are treated as independent. In this contribution we will investigate the possibilities of using multiantenna (i.e., triple and quadruple) data, not only for attitude determination but also for relative positioning. The methods developed are rigorous and have the additional advantage that they improve ambiguity resolution on the unconstrained baseline(s) and the overall success rate of ambiguity resolution between a number of antennas.

Copyright ( $) 2009$ Peter J. Buist et al. This is an open access article distributed under the Creative Commons Attribution License, which permits unrestricted use, distribution, and reproduction in any medium, provided the original work is properly cited.

\section{Introduction}

In this paper we explore methods for the combination of relative positioning and attitude determination for moving platforms, where each platform has multiantennas with known baseline lengths on its own surface and baseline vectors with unknown length to the other platforms. The objective of this research is to develop a method that optimally makes use of all the information available (i.e., the integerness of the ambiguities, the relationship between the ambiguities on the different baselines, and the known baseline length of the constrained baselines) to determine the relative position and orientation of a multiantenna system with unconstrained and constrained baselines. We develop a rigorous integrated method and investigate its ambiguity resolution performance for the unconstrained baselines and the overall success rate of the ambiguity resolution between a number of antennas. The paper begins with a discussion of potential applications and a literature review of previous work that has been done in this field. In Section 2 a general model for unconstrained and constrained baselines is introduced. Section 3 describes the standard methods for ambiguity resolution for unconstrained (e.g., relative navigation) and constrained (e.g., attitude determination) baseline applications. Section 4 introduces three methods for multiantenna ambiguity resolution and describes the methods mathematically for triple and quadruple antenna configurations. In Section 5 the methods are tested using simulated data.

\subsection{Applications}

1.1.1. Relative Navigation. Currently precise relative navigation using GNSS is under development for a large number of applications on land, on water, in the air, and even in space. The automotive industry shows interest in this application for relative navigation not only between vehicles and reference stations but also between vehicles [1]. Maritime applications, especially inshore relative navigation, require precise and robust methods [2]. Obviously this kind of technique not only is required for a swarm of Unmanned Aerial Vehicles (UAVs) [3, 4] or spacecraft [5] but also could be beneficial for swarms of manned vehicles [6]. Other aircraft applications are aerial refueling as well as, potentially, landing [7]. For relative navigation between aircraft and vessels, landing on aircraft carriers is an important application [8]. If the vehicles have multiple antennas, GNSS could potentially be used for determination of the attitude of the vehicle(s) [9-11]. Traditionally the relative positioning and attitude determination problems are treated as independent. In this contribution we investigate the possibility of using multiantenna data, not only for attitude determination but also to improve the relative positioning. 
1.1.2. Absolute and Relative Attitude Determination. Attitude determination using GNSS signals is becoming more and more accepted for real world applications. With 2 antennas/1 baseline, a direction estimate similar to a magnetic compass can be obtained. With 3 antennas/2 baselines, placed at appropriate relative positions, the full attitude can be determined. For some applications we would like to know the relative attitude between two platforms, which also could be provided by GNSS if both platforms have a number of antennas. Examples of these applications are not only aerial refueling, landing on aircraft carriers and rendezvous and docking in space but also formation flying if the elements of the formation have to point in certain directions relative to each other.

1.2. Previous Work. In [12] the use of a quadruple receiver system consisting of two static GPS receivers and two GPS receivers mounted on a single platform was considered for improved On The Fly (OTF) ambiguity resolution with single frequency receivers. The ambiguities between the two static receivers and between the two receivers on the same platform could be determined within a few seconds due to the short and fixed baselines between them. These ambiguities could, in turn, be used as constraints to reduce the number of potential ambiguity solutions for the unconstrained baseline between the static station and the platform and, therefore, to reduce the time to resolution from 810 to about 470 seconds for a configuration without choke rings and from 355 to 180 seconds for a configuration with choke rings. The research used the relationship between the ambiguities but did not model the correlation between the observations at the antennas.

In [13] a system was proposed which provides carrierbased positioning and two axis attitude measurements using three single frequency GPS receivers (i.e., triple-antenna configuration). The aim of this triple-antenna configuration was to increase the success rate of the integer ambiguity resolution process when relative positioning the platform to a base station by utilising knowledge of the integer ambiguities obtained from a constrained baseline in the attitude determination system. The use of baseline length or geometry constraints in the attitude determination environment increased the integer ambiguity success rate. In that paper the knowledge of the integer ambiguities from the attitude determination system is used to reduce the number of candidates during the search for the integer ambiguities arising when the third receiver is included. When these ambiguities are resolved, the unknown baselines between the roving (attitude) receivers and base receiver may be determined and the relative position obtained. The relation between the work of [13] and this paper will be discussed in more detail later.

Also commercial products are starting to use multiantenna data in their relative positioning solutions. One example is the TRIUMPH-4X from JAVAD, which uses quadruple antennas at both the base station and rover to calculate Real Time Kinematic (RTK) solutions, in what they call cluster RTK [14]. As it is a commercial product no details about their processing strategy are available.

\section{Modelling}

2.1. Model for Unconstrained Baselines. Precise GNSS receivers make use of two types of observations: pseudorange and carrier phase. The pseudorange observations typically have an accuracy of decimeters, whereas carrier phase observations have accuracies up to millimeter level. The double difference (hereafter coined DD) observation equations can be written as a system of linearized observation equations [15]:

$$
E(y)=A a+B b, \quad D(y)=Q_{y},
$$

where $E$ is the mean or the expected value and $D$ is the variance or dispersion of $y, y$ is the vector of "observed minus computed" DD carrier phases and/or code observations of the order $m, a$ is the unknown vector of ambiguities of the order $n$ expressed in cycles rather than range to maintain their integer character, $b$ is the baseline vector, which is unknown for relative navigation applications but for which the length in attitude determination is known, $B$ is the geometry matrix containing normalized line-of-sight vectors, that is, a matrix containing DD direction cosines, and $A$ is a design matrix that links the data vector to the unknown vector $a$. In this paper the assumption is made that the antennas are close to each other and thus atmospheric effects can be neglected. The variance matrix of $y$ is given by the positive definite matrix $Q_{y}$ which is assumed to be known. As explained in [15], the least squares solution of the linear system of observation equations as introduced in (1) is obtained, using $\|\cdot \cdot\|_{Q_{y}}^{2}=(\cdot \cdot)^{T} Q_{y}^{-1}(\cdot)$, from

$$
\min _{a \in \mathbb{Z}^{n}, b \in \mathbb{R}^{3}}\|y-A a-B b\|_{Q_{y}}^{2} .
$$

2.2. Model for Constrained Baselines. For a baselineconstrained application, as, for example, GNSS-based attitude determination, we can make use of the knowledge that the length of the baseline is known and constant. Hence the baseline-constrained integer ambiguity resolution can make use of the standard GNSS model by adding the length constraint of the baseline $\|b\|_{I_{3}}=l$, where $l$ is known. The observation equations then become [16]

$$
\begin{gathered}
E(y)=A a+B b, \quad D(y)=Q_{y}, \\
\|b\|_{I_{3}}=l, \quad a \in \mathbb{Z}^{n}, \quad b \in \mathbb{R}^{3} .
\end{gathered}
$$

Then the least squares criterion reads

$$
\min _{a \in \mathbb{Z}^{n}, b \in \mathbb{R}^{3},\|b\|_{I_{3}}=l}\|y-A a-B b\|_{Q_{y}}^{2} .
$$

This least squares problem is coined a Quadratically Constrained Integer Least Squares (QC-ILSs) problem in [17].

\section{Ambiguity Resolution}

High-precision positioning and attitude determination require the use of the very precise GNSS carrier phase observations, which however are ambiguous by an unknown integer number of cycles. For ambiguity resolution we make use 
of the LAMBDA (Least-squares AMBiguity Decorrelation Adjustment) method and its recently developed baselineconstrained extension [16]. These methods will briefly be discussed. A large number of ambiguity resolution techniques have been developed for the attitude determination application, as, for example, [18-26]. These are discussed in more detail in [27]. In this publication we focus on the standard and the constrained LAMBDA method but the proposed combination of relative positioning and attitude determination should also work with the other ambiguity resolution techniques.

3.1. The Standard LAMBDA Method. The least squares criterion for the unconstrained problem reads as $[15,28]$

$$
\begin{aligned}
& \min _{a \in \mathbb{Z}^{n}, b \in \mathbb{R}^{3}}\|y-A a-B b\|_{Q_{y}}^{2} \\
& \quad=\|\hat{e}\|_{Q_{y}}^{2}+\min _{a \in \mathbb{Z}^{n}, b \in \mathbb{R}^{3}}\left(\|\hat{a}-a\|_{Q_{\hat{a}}}^{2}+\|\hat{b}(a)-b\|_{Q_{\hat{b}(a)}}^{2}\right),
\end{aligned}
$$

where $\hat{e}=y-A \hat{a}-B \hat{b}$ is the least squares residual of the float solution $\hat{a}, \hat{b}$, and $\hat{b}(a)$ is the least squares solution for $b$, assuming that $a$ is known and $Q_{\hat{b}(a)}=Q_{\hat{b}}-Q_{\hat{b} \hat{a}} Q_{\hat{a}}^{-1} Q_{\hat{a} \hat{b}}$. The last term of (5) can be made zero for any $a$. We solve the vector of integer least-squares estimates of the ambiguities $a$ :

$$
\check{a}=\arg \left(\min _{a \in \mathbb{Z}^{n}}\|\hat{a}-a\|_{Q_{\hat{a}}}^{2}\right),
$$

where $\check{a}$ is the vector of integers that minimize the term within the brackets (arg or argument). A so-called integer search is needed to find $a$. The search space for this problem is defined as

$$
\Psi\left(\chi^{2}\right)=\left\{a \in \mathbb{Z}^{n} \mid\|\hat{a}-a\|_{Q_{\hat{a}}}^{2} \leq \chi^{2}\right\},
$$

where $\chi^{2}$ is a properly chosen constant. The LAMBDA method is an efficient way to find the minimizer of (6) [2931].

Once the solution $\check{a}$ has been obtained, the residual $(\hat{a}-$ $\check{a})$ is used to adjust the float solution $\hat{b}$ of the first step, and therefore the final fixed baseline solution is obtained as $\breve{b}=$ $\hat{b}(\check{a})=\hat{b}-Q_{\hat{b} \hat{a}} Q_{\hat{a}}^{-1}(\hat{a}-\check{a})$.

3.2. Baseline-Constrained LAMBDA Method. The least squares criterion for (4) of the baseline-constrained problem reads as

$$
\begin{aligned}
& \min _{\substack{a \in \mathbb{Z}^{n} \\
b \in \mathbb{R}^{3} \\
\|b\|=l}}\|y-A a-B b\|_{Q_{y}}^{2} \\
& \quad=\|\hat{e}\|_{Q_{y}}^{2}+\min _{a \in \mathbb{Z}^{n}}\left(\|\hat{a}-a\|_{Q_{\hat{a}}}^{2}+\min _{\substack{b \in \mathbb{R}^{3} \\
\|b\| l}}\left(\|\hat{b}(a)-b\|_{Q_{\hat{b}(a)}}^{2}\right)\right) .
\end{aligned}
$$

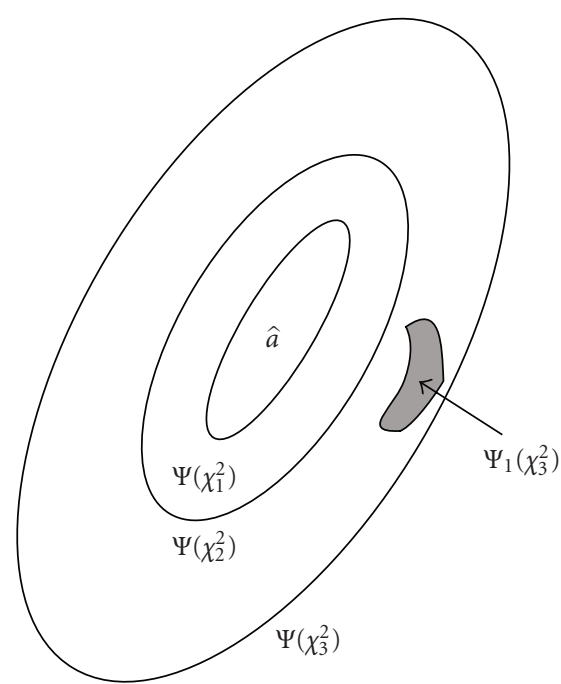

Figure 1: Baseline-constrained LAMBDA using the "Expansion approach."

In the constrained approach we will search for the integer least-squares ambiguity vector in the search space:

$$
\Psi_{1}\left(\chi^{2}\right)=\left\{a \in \mathbb{Z}^{n} \mid\|\hat{a}-a\|_{Q_{\hat{a}}}^{2}+\|\hat{b}(a)-\check{b}(a)\|_{Q_{\hat{b}(a)}}^{2} \leq \chi^{2}\right\},
$$

where $\check{b}(a)$ is the fixed solution for $b$, assuming that $a$ is known: $\check{b}(a)=\arg \left(\min _{b \in \mathbb{R}^{n},\|b\|=l}\|\hat{b}(a)-b\|_{Q_{\hat{b}(a)}}^{2}\right)$. The method applied in this contribution, and in $[27,32]$, is referred to as "Expansion approach." In the Expansion approach, we first use the standard LAMBDA method to collect integer vectors inside the search space $\Psi\left(\chi^{2}\right)$ and store all those that fulfill the inequality:

$$
\|\hat{b}(a)-\check{b}(a)\|_{Q_{\hat{b}(a)}}^{2} \leq \chi^{2}-\|\hat{a}-a\|_{Q_{\hat{a}}}^{2} .
$$

The initial search space is defined as the value $\chi_{1}^{2}=$ $\left\|\hat{a}-\check{a}_{B}\right\|_{Q_{\hat{a}}}^{2}$ where $\check{a}_{B}$ is the bootstrapped solution of $a$ $[15,29]$. This initial value $\chi_{1}^{2}$ is increased $k$ times until the search space $\Psi_{1}\left(\chi_{k}^{2}\right)$ is nonempty, using the logic visualized in Figure 1. For every step we enumerate all the integer vectors contained in $\Psi_{1}\left(\chi_{k}^{2}\right)$. If the set is nonempty, we pick up the minimizer; otherwise we increase $\chi_{k}^{2}$ and thus the size of the search space $\Psi_{1}\left(\chi_{k}^{2}\right)$.

For completeness we would like to mention that another method, the so-called "Search and Shrink approach," was developed to solve the same problem [33].

\section{Baseline-Constrained Multiantenna Ambiguity Resolution}

Precise relative positioning of two moving platforms usually requires dual-frequency phase data, whereas-due to the 


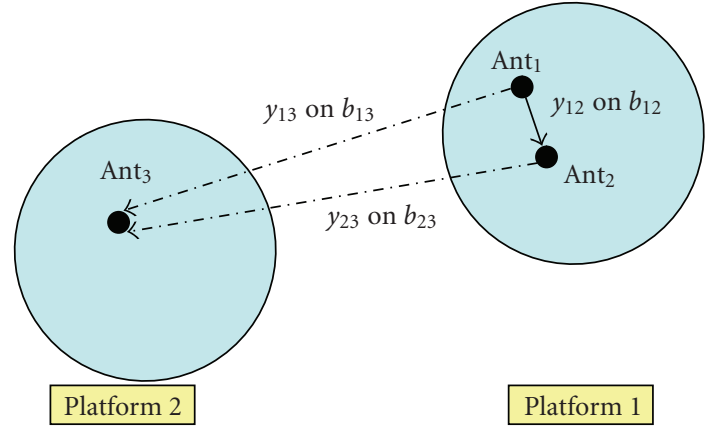

Figure 2: Definition of the triple-antenna configuration (solid arrows indicate baseline with known length).

baseline length constraints-single-frequency phase data may suffice for the precise determination of platform attitudes [5, 27, 32]. These two GNSS problems, relative positioning and attitude determination, are usually treated separately and independent from one another. In this contribution we combine the two into a multiantenna ambiguity resolution problem of which some of the baseline lengths are constrained. Insight in the numerical and statistical properties of these different approaches will be given. First we will introduce a 3- or triple- and 4or quadruple-antenna configuration, which we will use to investigate the processing strategies theoretically. These triple- and quadruple-antenna configurations are simplified models that represent experiments as described in $[5,27,34,35]$.

4.1. Multibaseline Setup. Consider three or four antennas on two platforms as shown in Figures 2 and 3, respectively. The baselines between antenna $j\left(\right.$ Ant $\left._{j}\right)$ and the antennas $i\left(\right.$ Ant $\left._{i}\right)$ are called baseline $i j\left(b_{i j}\right)$. The unconstrained baselines between an antenna at one platform and the antennas onboard another platform are $b_{23}, b_{13}$, and $b_{24}$ and the constrained baselines are baseline $12\left(b_{12}\right)$ and baseline $34\left(b_{34}\right)$ with lengths $l_{12}$ and $l_{34}$, respectively. The antennas are assumed to be sufficiently close, an assumption generally acceptable for the kind of applications discussed in Section 1.1, so that the relative antenna-satellite geometry may be considered the same for all antennas. The design matrices $A$ and $B$ and the variance-covariance matrix $Q_{y}$ are assumed to be identical. We take the ordering of the four antenna pairs such that $y_{i j}$ is the difference of the singledifferenced data of antenna $j$ minus that of antenna $i$.

\subsection{Model and Unconstrained Float Solution}

4.2.1. Triple-Antenna Configuration. For an integrated approach, we can use the known relationship between constrained and unconstrained baselines. For constrained baseline $b_{12}$ and unconstrained baselines $b_{23}$ and $b_{13}$, respectively, with common antennas we have the following relationship for the baseline, DD ambiguities, and DD

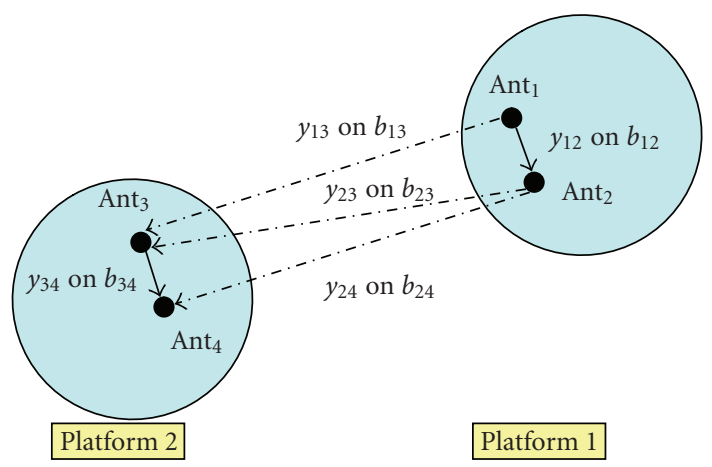

FIGURE 3: Definition of the quadruple-antenna configuration (solid arrows indicate baseline with known length).

observation vectors:

$$
\begin{aligned}
& b_{13}=b_{12}+b_{23}, \\
& a_{13}=a_{12}+a_{23}, \\
& y_{13}=y_{12}+y_{23} .
\end{aligned}
$$

This equation shows that two out of three DD data vectors are sufficient to set up the GNSS model.

For the 3 -antenna configuration, if we use $y_{12}$ and $y_{23}$, the model becomes

$$
\begin{aligned}
E\left[\begin{array}{l}
y_{12} \\
y_{23}
\end{array}\right] & =\left[\begin{array}{llll}
A & B & 0 & 0 \\
0 & 0 & A & B
\end{array}\right]\left[\begin{array}{l}
a_{12} \\
b_{12} \\
a_{23} \\
b_{23}
\end{array}\right], \\
D\left[\begin{array}{l}
y_{12} \\
y_{23}
\end{array}\right] & =\left[\begin{array}{ccc}
-1 & 1 & 0 \\
0 & -1 & 1
\end{array}\right]\left[\begin{array}{ccc}
\frac{1}{2} Q_{y} & 0 & 0 \\
0 & \frac{1}{2} Q_{y} & 0 \\
0 & 0 & \frac{1}{2} Q_{y}
\end{array}\right]\left[\begin{array}{cc}
-1 & 0 \\
1 & -1 \\
0 & 1
\end{array}\right] \\
& =\left[\begin{array}{cc}
Q_{y} & -\frac{1}{2} Q_{y} \\
-\frac{1}{2} Q_{y} & Q_{y}
\end{array}\right] .
\end{aligned}
$$

Note the presence of the nonzero covariance matrix $C\left(y_{12}, y_{23}\right)=-(1 / 2) Q_{y}$, which is due to the fact that the DD vectors $y_{12}$ and $y_{23}$ have an antenna in common.

Applying $P_{2}=\left[\begin{array}{cc}1 & -1 / 2 \\ -1 / 2 & 1\end{array}\right]$ and the Kronecker product $($ or symbol $) \otimes$ gives the following model:

$$
E\left[\begin{array}{l}
y_{12} \\
y_{23}
\end{array}\right]=I_{2} \otimes(A, B)\left[\begin{array}{l}
a_{12} \\
b_{12} \\
a_{23} \\
b_{23}
\end{array}\right], \quad D\left[\begin{array}{l}
y_{12} \\
y_{23}
\end{array}\right]=P_{2} \otimes Q_{y} .
$$


For a complete reference on the properties of the Kronecker product we refer to [36]. Now the least squares solution and corresponding variance matrix of the 3-antenna configuration can be given as

$$
\begin{gathered}
{\left[\begin{array}{l}
\hat{a}_{12} \\
\hat{b}_{12} \\
\hat{a}_{23} \\
\hat{b}_{23}
\end{array}\right]=\left[I_{2} \otimes\left(\left[(A, B)^{T} Q_{y}^{-1}(A, B)\right]^{-1}(A, B)^{T} Q_{y}^{-1}\right)\right]\left[\begin{array}{l}
y_{12} \\
y_{23}
\end{array}\right],} \\
D\left[\begin{array}{l}
\hat{a}_{12} \\
\hat{b}_{12} \\
\hat{a}_{23} \\
\hat{b}_{23}
\end{array}\right]=P_{2} \otimes\left[(A, B)^{T} Q_{y}^{-1}(A, B)\right]^{-1} .
\end{gathered}
$$

This shows that $\hat{a}_{i j}$ and $\hat{b}_{i j}$ are solely determined by the $\mathrm{DD}$ vector of the corresponding antenna pair, that is, $y_{i j}$, thus parallel processing is possible for the float solution. In Section 4.3 , it will be demonstrated that this property is lost once the integer constraints are applied. If we denote the variance-covariance matrix of $\hat{a}_{i j}$ and $\hat{b}_{i j}$ as

$$
\left[(A, B)^{T} Q_{y}^{-1}(A, B)\right]^{-1}=\left[\begin{array}{cc}
Q_{\hat{a}} & Q_{\hat{a} \hat{b}} \\
Q_{\hat{b} \hat{a}} & Q_{\hat{b}}
\end{array}\right],
$$

then the dispersion of the 3-antenna model can also be written as

$$
D\left[\begin{array}{l}
\hat{a}_{12} \\
\hat{b}_{12} \\
\hat{a}_{23} \\
\hat{b}_{23}
\end{array}\right]=\left[P_{2} \otimes\left[\begin{array}{cc}
Q_{\hat{a}} & Q_{\hat{a} \hat{b}} \\
Q_{\hat{b} \hat{a}} & Q_{\hat{b}}
\end{array}\right]\right]
$$

or after reordering

$$
D\left[\begin{array}{l}
\hat{a}_{12} \\
\hat{a}_{23} \\
\hat{b}_{12} \\
\hat{b}_{23}
\end{array}\right]=\left[\begin{array}{cc}
P_{2} \otimes Q_{\hat{a}} & P_{2} \otimes Q_{\hat{a} \hat{b}} \\
P_{2} \otimes Q_{\hat{b} \hat{a}} & P_{2} \otimes Q_{\hat{b}}
\end{array}\right] .
$$

If one wants to determine $a_{13}$ and $b_{13}$ from the above results it can be obtained from (see (11))

$$
\left[\begin{array}{l}
\hat{a}_{13} \\
\hat{b}_{13}
\end{array}\right]=\left[(1,1) \otimes\left[\begin{array}{ll}
I_{n} & 0 \\
0 & I_{3}
\end{array}\right]\right]\left[\begin{array}{l}
\hat{a}_{12} \\
\hat{b}_{12} \\
\hat{a}_{23} \\
\hat{b}_{23}
\end{array}\right] .
$$

Application of the variance propagation law shows that both the integer and baseline solutions on this baseline have the same precision as the integer and baseline solutions at the other baselines:

$$
\begin{aligned}
D\left[\begin{array}{l}
\hat{a}_{13} \\
\hat{b}_{13}
\end{array}\right]= & {\left[(1,1) \otimes\left[\begin{array}{cc}
I_{n} & 0 \\
0 & I_{3}
\end{array}\right]\right]\left[P_{2} \otimes\left[\begin{array}{cc}
Q_{\hat{a}} & Q_{\hat{a} \hat{b}} \\
Q_{\hat{b} \hat{a}} & Q_{\hat{b}}
\end{array}\right]\right] } \\
& \times\left[(1,1) \otimes\left[\begin{array}{ll}
I_{n} & 0 \\
0 & I_{3}
\end{array}\right]\right]^{T} \\
= & {\left[\begin{array}{ll}
Q_{\hat{a}} & Q_{\hat{a} \hat{b}} \\
Q_{\hat{b} \hat{a}} & Q_{\hat{b}}
\end{array}\right] . }
\end{aligned}
$$

4.2.2. Quadruple-Antenna Configuration. For constrained baselines $b_{12}$ and $b_{34}$ and unconstrained baselines $b_{23}, b_{13}$, and $b_{24}$, respectively, with common antennas we have the following relationship for the baseline, ambiguities, and observation vectors:

$$
\begin{aligned}
& b_{13}=b_{12}+b_{23}, \\
& a_{13}=a_{12}+a_{23}, \\
& y_{13}=y_{12}+y_{23}, \\
& b_{24}=b_{23}+b_{34}, \\
& a_{24}=a_{23}+a_{34}, \\
& y_{24}=y_{23}+y_{34} .
\end{aligned}
$$

This equation shows that now three out of five double difference data vectors are sufficient to set up the GNSS model.

Using the Kronecker symbol we can write also this model in a more compact form:

$$
E\left[\begin{array}{l}
y_{12} \\
y_{23} \\
y_{34}
\end{array}\right]=I_{3} \otimes(A, B)\left[\begin{array}{l}
a_{12} \\
b_{12} \\
a_{23} \\
b_{23} \\
a_{34} \\
b_{34}
\end{array}\right], \quad D\left[\begin{array}{l}
y_{12} \\
y_{23} \\
y_{34}
\end{array}\right]=P_{3} \otimes Q_{y}
$$

with

$$
P_{3}=\left[\begin{array}{ccc}
1 & -\frac{1}{2} & 0 \\
-\frac{1}{2} & 1 & -\frac{1}{2} \\
0 & -\frac{1}{2} & 1
\end{array}\right]
$$


The dispersion of the quadruple-antenna model can again be written as

$$
D\left[\begin{array}{l}
\hat{a}_{12} \\
\hat{b}_{12} \\
\hat{a}_{23} \\
\hat{b}_{23} \\
\hat{a}_{34} \\
\hat{b}_{34}
\end{array}\right]=\left[P_{3} \otimes\left[\begin{array}{cc}
Q_{\hat{a}} & Q_{\hat{a} \hat{b}} \\
Q_{\hat{b} \hat{a}} & Q_{\hat{b}}
\end{array}\right]\right]
$$

or again after reordering

$$
D\left[\begin{array}{l}
\hat{a}_{12} \\
\hat{a}_{23} \\
\hat{a}_{34} \\
\hat{b}_{12} \\
\hat{b}_{23} \\
\hat{b}_{34}
\end{array}\right]=\left[\begin{array}{cc}
P_{3} \otimes Q_{\hat{a}} & P_{3} \otimes Q_{\hat{a} \hat{b}} \\
P_{3} \otimes Q_{\hat{b} \hat{a}} & P_{3} \otimes Q_{\hat{b}}
\end{array}\right] .
$$

If one wants to determine $a_{13}, a_{24}$ and $b_{13}, b_{24}$ from the above results, it can be obtained from

\subsection{Optimal Solution of the Fully Integrated Approach}

4.3.1. Triple-Antenna Configuration. For the derivation of the integer least squares solution, which is the optimal solution, we use the 3-antenna configuration introduced in Section 4.1, for which the baseline $b_{12}$ is constrained and the baseline $b_{23}$ is unconstrained. First we write the sum-ofsquares decomposition as

$$
\begin{aligned}
\left\|\begin{array}{l}
y_{12}-A a_{12}-B b_{12} \\
y_{23}-A a_{23}-B b_{23}
\end{array}\right\|_{P_{2} \otimes Q_{y}}^{2}= & \left\|\begin{array}{l}
\hat{e}_{12} \\
\hat{e}_{23}
\end{array}\right\|_{P_{2} \otimes Q_{y}}^{2}+\left\|\begin{array}{l}
\hat{a}_{12}-a_{12} \\
\hat{a}_{23}-a_{23}
\end{array}\right\|_{P_{2} \otimes Q_{\hat{a}}}^{2} \\
& +\left\|\begin{array}{l}
\hat{b}_{12}\left(a_{12}, a_{23}\right)-b_{12} \\
\hat{b}_{23}\left(a_{12}, a_{23}\right)-b_{23}
\end{array}\right\|_{P_{2} \otimes Q_{\hat{b}(a)}}^{2} .
\end{aligned}
$$

The ambiguity-constrained baseline solution with variancecovariance matrix is given as

$$
\begin{aligned}
& {\left[\begin{array}{l}
\hat{b}_{12}\left(a_{12}, a_{23}\right) \\
\hat{b}_{23}\left(a_{12}, a_{23}\right)
\end{array}\right]} \\
& =\left[\begin{array}{l}
\hat{b}_{12} \\
\hat{b}_{23}
\end{array}\right]-\left(P_{2} \otimes Q_{\hat{b} \hat{a}}\right)\left(P_{2} \otimes Q_{\hat{a}}\right)^{-1}\left[\begin{array}{l}
\hat{a}_{12}-a_{12} \\
\hat{a}_{23}-a_{23}
\end{array}\right] \\
& =\left[\begin{array}{l}
\hat{b}_{12} \\
\hat{b}_{23}
\end{array}\right]-I_{2} \otimes Q_{\hat{b} \hat{a}} Q_{\hat{a}}^{-1}\left[\begin{array}{l}
\hat{a}_{12}-a_{12} \\
\hat{a}_{23}-a_{23}
\end{array}\right] \\
& =\left[\begin{array}{l}
\hat{b}_{12}\left(a_{12}\right) \\
\hat{b}_{23}\left(a_{23}\right)
\end{array}\right], \\
& D\left[\begin{array}{l}
\hat{b}_{12}\left(a_{12}, a_{23}\right) \\
\hat{b}_{23}\left(a_{12}, a_{23}\right)
\end{array}\right]=P_{2} \otimes Q_{\hat{b}(a)} .
\end{aligned}
$$

Therefore we can conclude that knowledge about $a_{12}$ does not improve the conditional baseline $\hat{b}_{23}\left(a_{23}\right)$, and similarly, knowledge about $a_{23}$ does not help to improve $\hat{b}_{12}\left(a_{12}\right)$. This is as expected from (12) assuming that the integers are known.

In order to obtain the unknown parameters we need to solve the following minimization problem:

$$
\begin{aligned}
& F\left(a_{12}, a_{23}, b_{12}, b_{23}\right) \\
& =\min _{\substack{a_{12}, a_{23} \in \mathbb{Z}^{n} \\
b_{12}, b_{23} \in \mathbb{R}^{3} \\
\left\|b_{12}\right\|_{I_{3}}=l}}\left\|\begin{array}{l}
y_{12}-A a_{12}-B b_{12} \\
y_{23}-A a_{23}-B b_{23}
\end{array}\right\|_{P_{2} \otimes Q_{y}}^{2} \\
& =\left\|\begin{array}{l}
\hat{e}_{12} \\
\hat{e}_{23}
\end{array}\right\|_{P_{2} \otimes Q_{y}}^{2} \\
& +\min _{\substack{a_{12}, a_{23} \in \mathbb{Z}^{n} \\
b_{12}, b_{23} \in \mathbb{R}^{3} \\
\left\|b_{12}\right\|_{I_{3}}=l_{12}}}\left(\left\|\begin{array}{l}
\| \hat{a}_{12}-a_{12} \\
\hat{a}_{23}-a_{23}
\end{array}\right\|_{P_{2} \otimes Q_{\hat{a}}}^{2}+\left\|\begin{array}{l}
\hat{b}_{12}\left(a_{12}\right)-b_{12} \\
\hat{b}_{23}\left(a_{23}\right)-b_{23}
\end{array}\right\|_{P_{2} \otimes Q_{\hat{b}(a)}}^{2}\right) .
\end{aligned}
$$

The last term on the right-hand side can be rewritten as

$$
\begin{aligned}
& \left\|\begin{array}{l}
\| \hat{b}_{12}\left(a_{12}\right)-b_{12} \\
\hat{b}_{23}\left(a_{23}\right)-b_{23}
\end{array}\right\|_{P_{2} \otimes Q_{\hat{b}(a)}}^{2} \\
& \quad=\left\|\hat{b}_{12}\left(a_{12}\right)-b_{12}\right\|_{Q_{\hat{b}(a)}}^{2}+\left\|\hat{b}_{23}\left(a_{23}, b_{12}\right)-b_{23}\right\|_{(3 / 4) Q_{\hat{b}(a)}}^{2} .
\end{aligned}
$$


With the constraint on the baseline $b_{12}$ and the ambiguities, the conditional solution of the baseline $b_{23}$ becomes

$$
\begin{aligned}
& \hat{b}_{23}\left(a_{23}, b_{12}\right) \\
& =\hat{b}_{23}\left(a_{23}\right)-\left(-\frac{1}{2} Q_{\hat{b}(a)}\right)\left(Q_{\hat{b}(a)}\right)^{-1}\left(\hat{b}_{12}\left(a_{12}\right)-b_{12}\right) \\
& =\hat{b}_{23}\left(a_{23}\right)+\frac{1}{2}\left(\hat{b}_{12}\left(a_{12}\right)-b_{12}\right) .
\end{aligned}
$$

The variance for this ambiguity constrained baseline is $D\left(\hat{b}_{23}\left(a_{23}, b_{12}\right)\right)=(3 / 4) Q_{\hat{b}(a)}$, and hence the knowledge of the constrained baseline allows us to improve the precision of the ambiguity constrained baseline from $Q_{\hat{b}(a)}$ to $(3 / 4) Q_{\hat{b}(a)}$.

The integer least squares solution of (28) then becomes

$$
\begin{aligned}
{\left[\begin{array}{c}
\check{a}_{12} \\
\check{a}_{23}
\end{array}\right]=} & \arg \min _{a_{12}, a_{23} \in \mathbb{Z}^{n}}\left(\|\| \begin{array}{l}
\hat{a}_{12}-a_{12} \|_{\hat{a}_{23}}-a_{23} \\
\hat{a}_{P_{2} \otimes Q_{\hat{a}}}^{2}
\end{array}\right. \\
& \left.+\min _{\left\|b_{12}\right\|=l_{12}}\left(\left\|\hat{b}_{12}\left(a_{12}\right)-b_{12}\right\|_{Q_{\hat{b}(a)}}^{2}\right)\right), \\
\check{b}_{12}= & \arg \min _{\left\|b_{12}\right\|=l_{12}}\left(\left\|\hat{b}_{12}\left(\check{a}_{12}\right)-b_{12}\right\|_{Q_{\hat{b}(a)}}^{2}\right), \\
\check{b}_{23}= & \hat{b}_{23}\left(\check{a}_{23}, \check{b}_{12}\right) \\
= & \hat{b}_{23}\left(\check{a}_{23}\right)+\frac{1}{2}\left(\hat{b}_{12}\left(\check{a}_{12}\right)-\check{b}_{12}\right) ;
\end{aligned}
$$

for which the ambiguity vector can also be written as

$$
\begin{aligned}
{\left[\begin{array}{c}
\check{a}_{12} \\
\check{a}_{23}
\end{array}\right]=\arg \min _{a_{12}, a_{23} \in \mathbb{Z}^{n}}(} & \left\|\hat{a}_{12}-a_{12}\right\|_{Q_{\hat{a}}}^{2} \\
& +\min _{\left\|b_{12}\right\|=l_{12}}\left(\left\|\hat{b}_{12}\left(a_{12}\right)-b_{12}\right\|_{Q_{\hat{b}(a)}}^{2}\right) \\
& \left.+\left\|\hat{a}_{23}\left(a_{12}\right)-a_{23}\right\|_{(3 / 4) Q_{\hat{a}}}^{2}\right) .
\end{aligned}
$$

The first two terms of the right-hand side of the equation form the ambiguity objective function for the constrained baseline as described in Section 3.2 (see (8)). The third term is due to the correlation between the ambiguities at the two baselines, where $\hat{a}_{23}\left(a_{12}\right)=\hat{a}_{23}-\left(-(1 / 2) Q_{\hat{a}}\right)\left(Q_{\hat{a}}\right)^{-1}\left(\hat{a}_{12}-\right.$ $\left.a_{12}\right)=\hat{a}_{23}+(1 / 2)\left(\hat{a}_{12}-a_{12}\right)$. This term contributes to the optimal solution, but because of the low correlation we expect this contribution to be small.

The processing strategy makes use of the steps explained in Sections 3.1 and 3.2 of the standard and the baselineconstrained LAMBDA method. We use the baselineconstrained LAMBDA to enumerate the ambiguities of the constrained baseline $b_{12}$ in combination with ambiguity vectors for baseline $b_{23}$ using the correlation between the ambiguities on the two baselines. In the final step we will use (31) to find the integer least squares solution.
4.3.2. Quadruple-Antenna Configuration. For the quadrupleantenna configuration with a constrained baseline, $b_{12}$ and $b_{34}$, respectively, on both sides of the ambiguity constrained baseline $b_{23}$, we can write

$$
\begin{aligned}
\left\|\begin{array}{l}
\hat{b}_{12}\left(a_{12}\right)-b_{12} \\
\hat{b}_{23}\left(a_{23}\right)-b_{23} \\
\hat{b}_{34}\left(a_{34}\right)-b_{34}
\end{array}\right\|_{P_{3} \otimes Q_{\hat{b}(a)}}^{2}= & \left\|\hat{b}_{12}\left(a_{12}\right)-b_{12}\right\|_{Q_{\hat{b}(a)}}^{2} \\
& +\left\|\hat{b}_{23}\left(a_{23}, b_{12}, b_{34}\right)-b_{23}\right\|_{(1 / 2) Q_{\hat{b}(a)}}^{2} \\
& +\left\|\hat{b}_{34}\left(a_{34}\right)-b_{34}\right\|_{Q_{\hat{b}(a)}^{2} .}^{2}
\end{aligned}
$$

With the constraint on the baselines $b_{12}$ and $b_{34}$ and the ambiguities, the conditional solution of the baseline $b_{23}$ becomes

$$
\begin{aligned}
\hat{b}_{23}\left(a_{23}, b_{12}, b_{34}\right)= & \hat{b}_{23}\left(a_{23}\right)+\frac{1}{2}\left(\hat{b}_{12}\left(a_{12}\right)-b_{12}\right) \\
& +\frac{1}{2}\left(\hat{b}_{34}\left(a_{34}\right)-b_{34}\right) .
\end{aligned}
$$

The second term on the right-hand side of (33) can be made zero for every $a_{23}$, and therefore we can write the minimization problem as

$$
\min _{\substack{a_{12}, a_{23}, a_{34} \in \mathbb{Z}^{n} \\
b_{12}, b_{34} \in \mathbb{R}^{3},\left\|b_{12}\right\|_{I_{3}}=l_{12},\left\|b_{34}\right\|_{I_{3}}=l_{34}}}\left(\|\| \begin{array}{l}
\mid \hat{a}_{12}-a_{12} \\
\hat{a}_{23}-a_{23} \\
\hat{a}_{34}-a_{34}
\end{array}\left\|_{P_{3} \otimes Q_{\hat{a}}}^{2}+\right\| \begin{array}{l}
\hat{b}_{12}\left(a_{12}\right)-b_{12} \\
\hat{b}_{34}\left(a_{34}\right)-b_{34}
\end{array} \|_{I_{2} \otimes Q_{\hat{b}(a)}}^{2}\right) .
$$

The integer least squares solution becomes for the 4-antenna configuration

$$
\begin{gathered}
{\left[\begin{array}{c}
\check{a}_{12} \\
\check{a}_{23} \\
\check{a}_{34}
\end{array}\right]=\arg \min _{a_{12}, a_{23}, a_{34} \in \mathbb{Z}^{n}}\left(\left\|\begin{array}{l}
\| \hat{a}_{12}-a_{12} \\
\hat{a}_{23}-a_{23} \\
\hat{a}_{34}-a_{34}
\end{array}\right\|_{P_{3} \otimes Q_{\hat{a}}}^{2}\right.} \\
+\min _{\left\|b_{12}\right\|=l_{12}}\left(\left\|\hat{b}_{12}\left(a_{12}\right)-b_{12}\right\|_{Q_{\hat{b}(a)}}^{2}\right) \\
\left.+\min _{\left\|b_{34}\right\|=l_{34}}\left(\left\|\hat{b}_{34}\left(a_{34}\right)-b_{34}\right\|_{Q_{\hat{b}(a)}^{2}}^{2}\right)\right), \\
\check{b}_{12}=\arg \min _{\left\|b_{12}\right\|=l_{12}}\left(\left\|\hat{b}_{12}\left(\check{a}_{12}\right)-b_{12}\right\|_{Q_{\hat{b}(a)}}^{2}\right), \\
\check{b}_{34}=\arg \min _{\left\|b_{34}\right\|=l_{34}}\left(\left\|\hat{b}_{34}\left(\check{a}_{34}\right)-b_{34}\right\|_{Q_{\hat{b}(a)}}^{2}\right),
\end{gathered}
$$




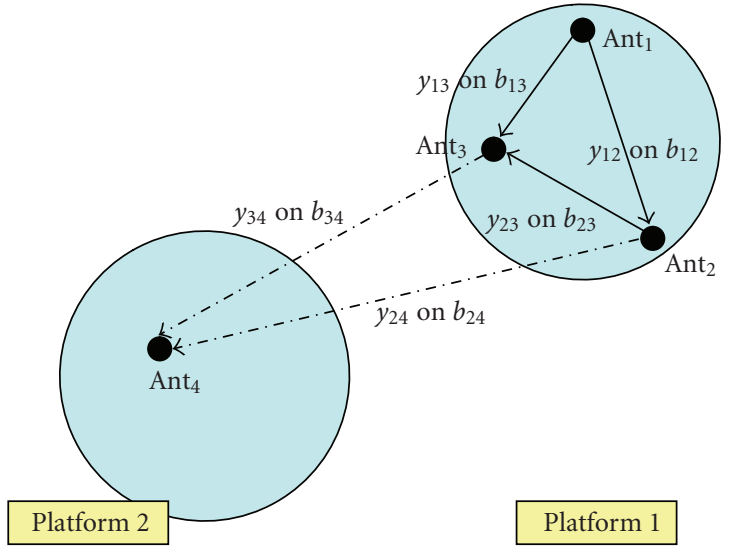

Figure 4: Definition of a different quadruple-antenna configuration (solid arrows indicate baseline with known length).

$$
\begin{aligned}
\check{b}_{23} & =\hat{b}_{23}\left(\check{a}_{23}, \check{b}_{12}, \check{b}_{34}\right) \\
& =\hat{b}_{23}\left(\check{a}_{23}\right)+\frac{1}{2}\left(\hat{b}_{12}\left(\check{a}_{12}\right)-\check{b}_{12}\right)+\frac{1}{2}\left(\hat{b}_{34}\left(\check{a}_{34}\right)-\check{b}_{34}\right) .
\end{aligned}
$$

Now the variance for this ambiguity-constrained baseline is $D\left(\hat{b}_{23}\left(a_{23}, b_{12}, b_{34}\right)\right)=(1 / 2) Q_{\hat{b}(a)}$, and hence the knowledge of 2 constrained baselines, one at each side of the unconstrained baseline, improves the precision of this baseline from $Q_{\hat{b}(a)}$ to $(1 / 2) Q_{\hat{b}(a)}$.

\subsubsection{Intermezzo: Alternative Quadruple-Antenna Configu-} ration. In Section 4.3.2 we have considered a quadrupleantenna configuration, assuming that we have two platforms, each with two antennas. The baseline lengths between the antennas on both platforms were assumed known $\left(b_{12}\right.$ and $b_{34}$ in Figure 3$)$. For completeness we assume now that we have a quadruple-antenna configuration with 3 antennas on one platform, again with known baseline lengths between the antennas, and 1 antenna on another platform. We will also give a solution for this configuration where both constrained baselines are placed at the same side of the unconstrained baseline (see Figure 4). For a quadrupleantenna configuration with the two constrained baselines $b_{12}$ and $b_{23}$ and the unconstrained baseline as $b_{34}$ we can write

$$
\begin{aligned}
\left\|\begin{array}{l}
\hat{b}_{12}\left(a_{12}\right)-b_{12} \\
\hat{b}_{23}\left(a_{23}\right)-b_{23} \\
\hat{b}_{34}\left(a_{34}\right)-b_{34}
\end{array}\right\|_{P_{3} \otimes Q_{\hat{b}(a)}}^{2}= & \left\|\hat{b}_{12}\left(a_{12}\right)-b_{12}\right\|_{Q_{\hat{b}(a)}}^{2} \\
& +\left\|\hat{b}_{23}\left(a_{23}, b_{12}\right)-b_{23}\right\|_{(3 / 4) Q_{\hat{b}(a)}}^{2} \\
& +\left\|\hat{b}_{34}\left(a_{34}, b_{12}, b_{23}\right)-b_{34}\right\|_{(2 / 3) Q_{\hat{b}(a)}}^{2}
\end{aligned}
$$

With the constraint on both the baselines $b_{12}$ and the ambiguities, the conditional solution of the baseline $b_{23}$ becomes

$$
\hat{b}_{23}\left(a_{23}, b_{12}\right)=\hat{b}_{23}\left(a_{23}\right)+\frac{1}{2}\left(\hat{b}_{12}\left(a_{12}\right)-b_{12}\right) .
$$

With the constraint on the baselines $b_{12}$ and $b_{23}$ and the ambiguities, the conditional solution of the baseline $b_{34}$ becomes

$$
\begin{aligned}
\hat{b}_{34}\left(a_{34}, b_{12}, b_{23}\right)= & \hat{b}_{34}\left(a_{34}\right)+\frac{1}{3}\left(\hat{b}_{12}\left(a_{12}\right)-b_{12}\right) \\
& +\frac{2}{3}\left(\hat{b}_{23}\left(a_{23}\right)-b_{23}\right) .
\end{aligned}
$$

The third term on the right-hand side of (37) can be made zero for every $a_{34}$, and therefore we can write the minimization problem, using the decomposition of (29), as

$$
\min _{\substack{a_{12}, a_{23}, a_{34} \in \mathbb{Z}^{n} \\
b_{12}, b_{23} \in \mathbb{R}^{3},\left\|b_{12}\right\|_{I_{3}}=l_{12},\left\|b_{23}\right\|_{I_{3}}=l_{23}}}\left(\left\|\begin{array}{l}
\hat{a}_{12}-a_{12} \\
\hat{a}_{23}-a_{23} \\
\hat{a}_{34}-a_{34}
\end{array}\right\|_{P_{3} \otimes Q_{\hat{a}}}^{2}+\| \begin{array}{l}
\hat{b}_{12}\left(a_{12}\right)-b_{12}\left\|_{\hat{b}_{23}\left(a_{23}\right)-b_{23}}\right\|_{P_{2} \otimes Q_{\hat{b}(a)}}^{2}
\end{array}\right) .
$$

The integer least squares solution becomes for this 4-antenna configuration

$$
\begin{aligned}
& {\left[\begin{array}{l}
\check{a}_{12} \\
\check{a}_{23} \\
\check{a}_{34}
\end{array}\right]} \\
& =\arg \min _{a_{12}, a_{23}, a_{34} \in \mathbb{Z}^{n}}\left(\left\|\begin{array}{l}
\hat{a}_{12}-a_{12} \\
\hat{a}_{23}-a_{23} \\
\hat{a}_{34}-a_{34}
\end{array}\right\|_{P_{3} \otimes Q_{\hat{a}}}^{2}\right. \\
& \left.+\min _{\substack{\left\|b_{12}\right\|_{I_{3}=l_{12}},\left\|b_{23}\right\|_{I_{3}}=l_{23}}}\left(\left\|\hat{b}_{12}\left(a_{12}\right)-b_{12}\right\|_{\hat{b}_{23}\left(a_{23}\right)-b_{23}}^{2} \|_{P_{2} \otimes Q_{\hat{b}(a)}}^{2}\right)\right), \\
& {\left[\begin{array}{c}
\check{b}_{12} \\
\check{b}_{23}
\end{array}\right]=\arg \min _{\substack{\left\|b_{12}\right\|_{I_{3}}=l_{12} \\
\left\|b_{23}\right\|_{I_{3}}=l_{23}}}\left(\left\|\hat{b}_{12}\left(a_{12}\right)-b_{12}\right\|_{\hat{b}_{23}\left(a_{23}\right)-b_{23}} \|_{P_{2} \otimes Q_{\hat{b}(a)}}^{2}\right),} \\
& \check{b}_{34}=\widehat{b}_{34}\left(\check{a}_{34}, \check{b}_{12}, \check{b}_{23}\right) \\
& =\widehat{b}_{34}\left(\check{a}_{34}\right)+\frac{1}{3}\left(\hat{b}_{12}\left(\check{a}_{12}\right)-\check{b}_{12}\right)+\frac{2}{3}\left(\hat{b}_{23}\left(\check{a}_{23}\right)-\check{b}_{23}\right) \text {. }
\end{aligned}
$$

Now the variance for this ambiguity constrained baseline is $D\left(\hat{b}_{34}\left(a_{34}, b_{12}, b_{23}\right)\right)=(2 / 3) Q_{\hat{b}(a)}$, and hence the knowledge of two constrained baselines, at the same side of the unconstrained baseline, improves the precision of the ambiguity constrained baseline from $Q_{\hat{b}(a)}$ to $(2 / 3) Q_{\hat{b}(a)}$. This means 
that the improvement is larger than for the triple-antenna configuration, as the model becomes stronger due to the two constrained baselines at one side, and less than the quadruple-antenna configuration of the previous section. This is also as expected as the unconstrained baseline is now only constrained at one side; the other side is left free.

\subsection{Suboptimal Solution of the Fully Integrated Approach}

4.4.1. Triple-Antenna Configuration. An approximation of the integer least squares solution as given in Section 4.3.1 can be obtained by first solving the first two terms on the righthand side of (32) for $a_{12}$, and then the third term for $a_{23}$, as if the correlation would be absent. The result is a vectorial bootstrapping approach in which we first solve the ambiguity on the constrained baseline and apply the found ambiguity vector in the solution of the unconstrained baseline. This is also the difference with the uncoupled approach from Section 4.5 in which the solutions are found completely independent of each other. This solution, hereafter coined the suboptimal solution, is then given as

$$
\begin{aligned}
& \check{a}_{12}=\arg \min _{a_{12} \in \mathbb{Z}^{n}}\left(\left\|\hat{a}_{12}-a_{12}\right\|_{Q_{\hat{a}}}^{2}\right. \\
& \left.\quad+\min _{\left\|b_{12}\right\|=l_{12}}\left(\left\|\hat{b}_{12}\left(a_{12}\right)-b_{12}\right\|_{Q_{\hat{b}(a)}}^{2}\right)\right), \\
& \check{a}_{23}=\arg \min _{a_{23} \in \mathbb{Z}^{n}}\left(\left\|\hat{a}_{23}\left(\check{a}_{12}\right)-a_{23}\right\|_{(3 / 4) Q_{\hat{a}}}^{2}\right), \\
& \check{b}_{12}=\arg \min _{\left\|b_{12}\right\|=l_{12}}\left(\left\|\hat{b}_{12}\left(\check{a}_{12}\right)-b_{12}\right\|_{Q_{\hat{b}(a)}}^{2}\right), \\
& \check{b}_{23}=\hat{b}_{23}\left(\check{a}_{23}\right)+\frac{1}{2}\left(\hat{b}_{12}\left(\check{a}_{12}\right)-\check{b}_{12}\right) .
\end{aligned}
$$

For this approach in the first step we use the baselineconstrained LAMBDA to estimate the ambiguities of the constrained baseline $b_{12}$. In the second step we use standard LAMBDA with $\hat{a}_{23}\left(\check{a}_{12}\right)$ and $(3 / 4) Q_{\hat{a}}$ on the unconstrained baseline. From (42), and (32), it is expected that the success rate of the integer least squares (optimal) approach is better than the vectorial bootstrapping (suboptimal) approach [37], but because of the low correlation between the two baselines the difference is anticipated to be minimal as discussed in the previous section. This is analyzed using simulated data in Section 5.

Next we will demonstrate that the suboptimal solution is the same as the solution from [13] in which the ambiguity vector for $b_{23}$ that minimizes the cost function in the metric of $Q_{\hat{a}}$ was found as

$$
\check{a}_{23}=\arg \min _{a_{23} \in \mathbb{Z}^{n}}\left(\left\|\hat{a}_{23}-a_{23}\right\|_{Q_{\hat{a}}}^{2}+\left\|\left(\hat{a}_{13}-a_{12}\right)-a_{23}\right\|_{Q_{\hat{a}}}^{2}\right) .
$$

We recall that we have obtained in Section 4.3.1 that $\hat{a}_{23}\left(a_{12}\right)=\hat{a}_{23}+(1 / 2)\left(\hat{a}_{12}-a_{12}\right)$, which we can rewrite using $\hat{a}_{12}=\hat{a}_{13}-\hat{a}_{23}$, as

$$
\hat{a}_{23}\left(a_{12}\right)=\frac{1}{2}\left(\hat{a}_{23}+\hat{a}_{13}-a_{12}\right) .
$$

Thus $\hat{a}_{23}\left(a_{12}\right)$ is the average of $\hat{a}_{23}$ and $\hat{a}_{13}-a_{12}$. Next we introduce the following identity, which is valid for any $u, v, x \in \mathbb{R}^{n}$ and arbitrary positive definite matrix $M$ :

$$
\left\|\frac{1}{2}(u+v)-x\right\|_{M}^{2}=\frac{1}{2}\|u-x\|_{M}^{2}+\frac{1}{2}\|v-x\|_{M}^{2}-\frac{1}{4}\|u-v\|_{M}^{2} .
$$

With this identity we obtain

$$
\begin{aligned}
\left\|\hat{a}_{23}\left(a_{12}\right)-a_{23}\right\|_{Q_{\hat{a}}}^{2}= & \left\|\frac{1}{2}\left(\hat{a}_{23}+\left(\hat{a}_{13}-a_{12}\right)-a_{23}\right)\right\|_{Q_{\hat{a}}}^{2} \\
= & \frac{1}{2}\left\|\hat{a}_{23}-a_{23}\right\|_{Q_{\hat{a}}}^{2} \\
& +\frac{1}{2}\left\|\left(\hat{a}_{13}-a_{12}\right)-a_{23}\right\|_{Q_{\hat{a}}}^{2} \\
& -\frac{1}{4}\left\|\hat{a}_{23}-\left(\hat{a}_{13}-a_{12}\right)\right\|_{Q_{\hat{a}} .}^{2}
\end{aligned}
$$

As the last term on the right-hand side is constant, we obtain the following solution:

$$
\begin{aligned}
\check{a}_{23} & =\arg \min _{a_{23} \in \mathbb{Z}^{n}}\left(\left\|\hat{a}_{23}\left(\check{a}_{12}\right)-a_{23}\right\|_{Q_{\hat{a}}}^{2}\right) \\
& =\arg \min _{a_{23} \in \mathbb{Z}^{n}}\left(\left\|\hat{a}_{23}-a_{23}\right\|_{Q_{\hat{a}}}^{2}+\left\|\left(\hat{a}_{13}-a_{12}\right)-a_{23}\right\|_{Q_{\hat{a}}}^{2}\right) .
\end{aligned}
$$

The first expression (i.e., (47a)) is the same as (42b) with $Q_{\hat{a}}$ instead of $(3 / 4) Q_{\hat{a}}$. This difference in scaling will not affect the outcome of the minimization. The second expression (i.e., see (47b)) is the one used in [13].

4.4.2. Quadruple-Antenna Configuration. An approximation of the integer least squares solution as given in Section 4.3.2 can again be obtained by solving the unconstrained baselines, after the constrained baselines are resolved. This would result in three minimizations, one for either $a_{12}$ and $a_{34}$ and one for $a_{23}$, if the correlation would be absent. The result is a vectorial bootstrapping approach in which we first solve the ambiguity on the constrained baselines $b_{12}$ and $b_{34}$ and apply the found ambiguity vector in the solution of the unconstrained baseline $a_{23}$. Hence the suboptimal solution for the 4-antenna configuration is given as

$$
\begin{aligned}
\check{a}_{12}=\arg \min _{a_{12} \in \mathbb{Z}^{n}} & \left(\left\|\hat{a}_{12}-a_{12}\right\|_{Q_{\hat{a}}}^{2}\right. \\
& \left.+\min _{\left\|b_{12}\right\|=l_{12}}\left(\left\|\hat{b}_{12}\left(a_{12}\right)-b_{12}\right\|_{Q_{\hat{b}(a)}}^{2}\right)\right), \\
\check{a}_{34}=\arg \min _{a_{34} \in \mathbb{Z}^{n}}\left(\left\|\hat{a}_{34}-a_{34}\right\|_{Q_{\hat{a}}}^{2}\right. & \\
& \left.+\min _{\left\|b_{34}\right\|=l_{34}}\left(\left\|\hat{b}_{34}\left(a_{34}\right)-b_{34}\right\|_{Q_{\hat{b}(a)}}^{2}\right)\right),
\end{aligned}
$$


TABLE 1: Simulation specification.

\begin{tabular}{|c|c|}
\hline Date and time & 22 Jan 2008 00:00 \\
\hline Location & Lat: $50^{\circ}$, Long: $3^{\circ}$ \\
\hline GPS week & 439 \\
\hline Scenario & Triple- and Quadruple-antenna, orthogonal configuration, stationary \\
\hline Frequency & L1 \\
\hline Number of satellites & $5-6-7-8$ \\
\hline Undifferenced code noise $\sigma_{p}[\mathrm{~cm}]$ & $30-15-5$ \\
\hline Undifferenced phase noise $\sigma_{\phi}[\mathrm{mm}]$ & $3-1$ \\
\hline Baseline length $\left\|b_{12}\right\|=\left\|b_{23}\right\|=\left\|b_{34}\right\|=l$ & $2.0 \mathrm{~m}$ \\
\hline Epochs simulated & $10^{5}$ \\
\hline
\end{tabular}

$$
\begin{aligned}
& \check{a}_{23}=\arg \min _{a_{23} \in \mathbb{Z}^{n}}\left(\left\|\hat{a}_{23}\left(\check{a}_{12}, \check{a}_{34}\right)-a_{23}\right\|_{(1 / 2) Q_{\hat{a}}}^{2}\right), \\
& \check{b}_{12}=\arg \min _{\left\|b_{12}\right\|=l_{12}}\left(\left\|\hat{b}_{12}\left(\check{a}_{12}\right)-b_{12}\right\|_{Q_{\hat{b}(a)}}^{2}\right) \\
& \check{b}_{34}=\arg \min _{\left\|b_{34}\right\|=l_{34}}\left(\left\|\hat{b}_{34}\left(\check{a}_{34}\right)-b_{34}\right\|_{Q_{\hat{b}(a)}}^{2}\right), \\
& \check{b}_{23}=\hat{b}_{23}\left(\check{a}_{23}\right)+\frac{1}{2}\left(\hat{b}_{12}\left(\check{a}_{12}\right)-\check{b}_{12}\right)+\frac{1}{2}\left(\hat{b}_{34}\left(\check{a}_{34}\right)-\check{b}_{34}\right) .
\end{aligned}
$$

For this approach in the first step we use the baselineconstrained LAMBDA to estimate the ambiguities of the two constrained baselines $b_{12}$ and $b_{34}$. In the second step we use standard LAMBDA with $\hat{a}_{23}\left(\check{a}_{12}, \check{a}_{34}\right)$ and $(1 / 2) Q_{\hat{a}}$ on the unconstrained baseline.

4.5. Uncoupled Approach Using Unconstrained and Constrained Baselines. The simplest way to combine constrained and unconstrained baselines is the uncoupled approach in which the baselines are treated completely independently. This approach provides a lower bound for the empirical success rate of the optimal and suboptimal approaches described in the previous two sections. This follows from the demonstration for these two approaches that the precision of the variance matrix of the ambiguities on the unconstrained baseline, evidently only if the success rate of the constrained baseline is close enough to 1 , was improved. For a discussion on lower bounds of the probability of obtaining the correct integer ambiguity vector, we refer to [37].

\section{Verification through Simulation}

In this section, the introduced approaches are applied, using simulated data, to the most challenging application of single epoch, single frequency ambiguity resolution. We will investigate the experimental or empirical success rate, which depends on the strength of the underlying GNSS model. For analysis of the performance of the described approaches, we compare the true integer ambiguity vector (the "true solution" known in the simulations) and the estimated integer ambiguity vector at every epoch. The empirical success rate is defined as the number of epochs where the obtained integer ambiguity vector was equal to the true integer ambiguity vector divided by the total number of epochs. The baseline length, as long as the atmospheric effects on the GPS observations are negligible (typically if the baseline is shorter than $10 \mathrm{~km}$, see [5]), will not influence the performance of the ambiguity resolution method. In this contribution we will simulate and analyze short baselines; however the results will also apply to longer baselines (between different platforms) as long as the atmospheric influences are small. We will analyze performance both on individual baselines and on the solution of combined baselines (the "overall" solution). The first result is important as the information of the constrained baseline could improve the solution of the unconstrained baseline, and furthermore we would like to confirm that the success rate for the constrained baseline is not changed in the integrated solution compared to the uncoupled solution. The second result is important, as for some applications as described in Section 1.1, we are interested in the estimation of a number of baseline vectors on or between a number of platforms.

5.1. Simulation Setup. In order to investigate the performance of the proposed approaches, we analyze the empirical success rates using simulated data. Table 1 summarizes the conditions of the simulations. Utilizing the VISUAL software [38], based on the location of the receivers and an actual GPS constellation, the design matrices of the model are calculated. In order to obtain good approximations, the number of samples must be sufficiently large [39]. Assuming different levels of noise on the undifferenced phase (from $1 \mathrm{~mm}$ to $3 \mathrm{~mm}$ ) and undifferenced code (from $5 \mathrm{~cm}$ to $30 \mathrm{~cm}$ ) data, a set of $10^{5}$ data was generated; then each simulation was repeated for different numbers of satellites varying between 5 and 8. Our choice for simulation parameters was to show the improvement of the integrated method compared to uncoupled. The subsets of GPS satellites were selected based on elevation angles.

5.2. Simulation Results. In this section we will analyze the proposed integrated approaches. For the suboptimal solution we observed a maximum difference in the empirical success rate with the optimal solution of $0.1 \%$; therefore there is only a marginal difference between the two and we include only the optimal solution's results in this paper. 
TABLE 2: Simulation results: single-frequency, single-epoch success rates for the constrained baseline $b_{12}$ using one constrained baseline ( $C=1$, i.e., two antennas for uncoupled and triple-antenna configuration for the optimal solution).

\begin{tabular}{|c|c|c|c|c|c|c|c|}
\hline \multirow[t]{2}{*}{$N_{S V}$} & \multirow[t]{2}{*}{$\# C$} & \multicolumn{3}{|c|}{$\begin{array}{c}\sigma_{\phi}[\mathrm{mm}]=3 \\
\sigma_{p}[\mathrm{~cm}]\end{array}$} & \multicolumn{3}{|c|}{$\begin{array}{c}\sigma_{\phi}[\mathrm{mm}]=1 \\
\sigma_{p}[\mathrm{~cm}]\end{array}$} \\
\hline & & 30 & 15 & 5 & 30 & 15 & 5 \\
\hline & 1 & \multicolumn{6}{|c|}{ Uncoupled $P\left(\tilde{a}_{12}=a_{12}\right)$} \\
\hline & 1 & \multicolumn{6}{|c|}{ Optimal $P\left(\check{a}_{12}=a_{12}\right)$} \\
\hline \multirow{2}{*}{5} & 1 & 0.72 & 0.89 & 1.00 & 0.97 & 1.00 & 1.00 \\
\hline & 1 & 0.72 & 0.89 & 1.00 & 0.97 & 1.00 & 1.00 \\
\hline \multirow{2}{*}{6} & 1 & 0.96 & 0.99 & 1.00 & 1.00 & 1.00 & 1.00 \\
\hline & 1 & 0.96 & 0.99 & 1.00 & 1.00 & 1.00 & 1.00 \\
\hline \multirow{2}{*}{7} & 1 & 0.99 & 1.00 & 1.00 & 1.00 & 1.00 & 1.00 \\
\hline & 1 & 1.00 & 1.00 & 1.00 & 1.00 & 1.00 & 1.00 \\
\hline \multirow{2}{*}{8} & 1 & 1.00 & 1.00 & 1.00 & 1.00 & 1.00 & 1.00 \\
\hline & 1 & 1.00 & 1.00 & 1.00 & 1.00 & 1.00 & 1.00 \\
\hline
\end{tabular}

TABLE 3: Simulation results: single-frequency, single-epoch success rates for the unconstrained baseline $b_{23}$ using zero $(C=0)$, one $(C=1$, i.e., triple-antenna configuration), or two $(C=2$, i.e., quadruple-antenna configuration) constrained baselines.

\begin{tabular}{|c|c|c|c|c|c|c|c|}
\hline \multirow[t]{2}{*}{$N_{S V}$} & \multirow[t]{2}{*}{$\# C$} & \multicolumn{3}{|c|}{$\begin{array}{c}\sigma_{\phi}[\mathrm{mm}]=3 \\
\sigma_{p}[\mathrm{~cm}]\end{array}$} & \multicolumn{3}{|c|}{$\begin{array}{c}\sigma_{\phi}[\mathrm{mm}]=1 \\
\sigma_{p}[\mathrm{~cm}]\end{array}$} \\
\hline & & 30 & 15 & 5 & 30 & 15 & 5 \\
\hline & 0 & \multicolumn{6}{|c|}{ Uncoupled $P\left(\tilde{a}_{23}=a_{23}\right)$} \\
\hline & 1 & \multicolumn{6}{|c|}{ Optimal $P\left(\check{a}_{23}=a_{23}\right)$} \\
\hline & 2 & \multicolumn{6}{|c|}{ Optimal $P\left(\check{a}_{23}=a_{23}\right)$} \\
\hline \multirow{3}{*}{5} & 0 & 0.03 & 0.19 & 0.87 & 0.06 & 0.27 & 0.95 \\
\hline & 1 & 0.04 & 0.26 & 0.93 & 0.09 & 0.36 & 0.98 \\
\hline & 2 & 0.09 & 0.42 & 0.98 & 0.15 & 0.52 & 1.00 \\
\hline \multirow{3}{*}{6} & 0 & 0.25 & 0.67 & 0.97 & 0.49 & 0.87 & 1.00 \\
\hline & 1 & 0.36 & 0.80 & 0.99 & 0.59 & 0.92 & 1.00 \\
\hline & 2 & 0.55 & 0.92 & 1.00 & 0.74 & 0.97 & 1.00 \\
\hline \multirow{3}{*}{7} & 0 & 0.50 & 0.80 & 1.00 & 0.75 & 0.93 & 1.00 \\
\hline & 1 & 0.61 & 0.89 & 1.00 & 0.81 & 0.97 & 1.00 \\
\hline & 2 & 0.74 & 0.96 & 1.00 & 0.89 & 0.99 & 1.00 \\
\hline \multirow{3}{*}{8} & 0 & 0.86 & 0.95 & 1.00 & 1.00 & 1.00 & 1.00 \\
\hline & 1 & 0.92 & 0.97 & 1.00 & 1.00 & 1.00 & 1.00 \\
\hline & 2 & 0.97 & 0.99 & 1.00 & 1.00 & 1.00 & 1.00 \\
\hline
\end{tabular}

We analyze the optimal solution using one constrained baseline (as in the triple-antenna configuration described in Section 4.3.1) and two constrained baselines (as in the quadruple-antenna configuration described in Section 4.3.2). The results are presented in three tables. Tables 2, 3, and 4 contain empirical success rates as a function of the number of tracked satellites $\left(N_{S V}\right)$ and the phase and code level noise $\left(\sigma_{\phi}, \sigma_{p}\right)$. In Tables 2 and 3 , we analyze the success rate on individual baselines, both uncoupled and as part of the optimal solution.

In these tables $P\left(\tilde{a}_{12}=a_{12}\right)$ and $P\left(\check{a}_{12}=a_{12}\right)$ stand for the success rate on the constrained baseline $b_{12}$ and $P\left(\tilde{a}_{23}=a_{23}\right)$ and $P\left(\check{a}_{23}=a_{23}\right)$ for the success rate on the unconstrained baseline $b_{23}$ in the uncoupled and optimal solution, respectively. For the unconstrained baseline we will analyze the uncoupled case $P\left(\tilde{a}_{23}=a_{23}\right)$, the case where one constrained baseline is utilized $(C=1)$ and the case where two constrained baselines are utilized $(C=2)$.

In Table 4, we look at the overall empirical success rate, which is the success rate on both baselines $\left(P\left(\check{a}_{12}=\right.\right.$ $\left.a_{12}, \check{a}_{23}=a_{23}\right)$ ) for the triple-antenna configuration or on all three baselines $\left(P\left(\check{a}_{12}=a_{12}, \check{a}_{23}=a_{23}, \check{a}_{34}=a_{34}\right)\right)$ for the quadruple-antenna configuration in a combined solution. Again the uncoupled and optimal approaches using one or two constrained baseline(s) are considered.

Uncoupled versus Optimal Solution. In Table 2, we observe that the solution on the constrained baseline, as part of the optimal solution, has the same performance as the uncoupled baseline-constrained solution.

In Table 3, the unconstrained baseline $b_{23}$ is presented as uncoupled $(C=0)$ and as part of the optimal solution 
TABLE 4: Simulation results: single-frequency, single-epoch overall success rates for two baselines or three baselines using zero $(C=0)$, one ( $C=1$, i.e., triple-antenna configuration $)$, or two $(C=2$, i.e., quadruple-antenna configuration) constrained baselines.

\begin{tabular}{|c|c|c|c|c|c|c|c|}
\hline \multirow[t]{2}{*}{$N_{S V}$} & \multirow[t]{2}{*}{$\# C$} & \multicolumn{3}{|c|}{$\begin{array}{c}\sigma_{\phi}[\mathrm{mm}]=3 \\
\sigma_{p}[\mathrm{~cm}]\end{array}$} & \multicolumn{3}{|c|}{$\begin{array}{c}\sigma_{\phi}[\mathrm{mm}]=1 \\
\sigma_{p}[\mathrm{~cm}]\end{array}$} \\
\hline & & 30 & 15 & 5 & 30 & 15 & 5 \\
\hline & 0 & \multicolumn{6}{|c|}{ Uncoupled $P\left(\tilde{a}_{12}=a_{12}, \check{a}_{23}=a_{23}\right)$} \\
\hline & 1 & \multicolumn{6}{|c|}{ Optimal $P\left(\check{a}_{12}=a_{12}, \check{a}_{23}=a_{23}\right)$} \\
\hline & 2 & \multicolumn{6}{|c|}{ Optimal $P\left(\check{a}_{12}=a_{12}, \check{a}_{23}=a_{23}, \check{a}_{34}=a_{34}\right)$} \\
\hline \multirow{3}{*}{5} & 0 & 0.02 & 0.17 & 0.86 & 0.05 & 0.27 & 0.95 \\
\hline & 1 & 0.04 & 0.25 & 0.93 & 0.09 & 0.36 & 0.98 \\
\hline & 2 & 0.06 & 0.38 & 0.98 & 0.15 & 0.52 & 1.00 \\
\hline \multirow{3}{*}{6} & 0 & 0.24 & 0.66 & 0.97 & 0.49 & 0.87 & 1.00 \\
\hline & 1 & 0.36 & 0.79 & 0.99 & 0.59 & 0.92 & 1.00 \\
\hline & 2 & 0.53 & 0.92 & 1.00 & 0.74 & 0.97 & 1.00 \\
\hline \multirow{3}{*}{7} & 0 & 0.50 & 0.80 & 1.00 & 0.75 & 0.93 & 1.00 \\
\hline & 1 & 0.61 & 0.89 & 1.00 & 0.81 & 0.97 & 1.00 \\
\hline & 2 & 0.74 & 0.96 & 1.00 & 0.89 & 0.99 & 1.00 \\
\hline \multirow{3}{*}{8} & 0 & 0.86 & 0.95 & 1.00 & 1.00 & 1.00 & 1.00 \\
\hline & 1 & 0.92 & 0.97 & 1.00 & 1.00 & 1.00 & 1.00 \\
\hline & 2 & 0.97 & 0.99 & 1.00 & 1.00 & 1.00 & 1.00 \\
\hline
\end{tabular}

using a single constrained baseline $(C=1)$ and using two constrained baselines $(C=2)$. As expected, when comparing Tables 2 and 3, the baseline-constrained solution clearly provides much better results than the unconstrained solution. The differences in success rate are particularly pronounced when the strength of the underlying GNSS model becomes weaker (fewer satellites and/or higher measurement noise). According to Table 2 already 5 satellites and a phase standard deviation of $3 \mathrm{~mm}$ give a higher than $70 \%$ success rate for the constrained solution.

For the unconstrained baseline in Table 3 we observe that the optimal solution has a better performance than uncoupled. The improvement is between $0 \%$ and $13 \%$ using a single constrained baseline $(C=1)$ and between $0 \%$ and $30 \%$ using two constrained baselines $(C=2)$, with a larger improvement for weaker GNSS models.

In Table 4 compared to the uncoupled approach $(C=0)$, the improvement of the empirical success rate for the optimal solution is between $0 \%$ and $13 \%$ if a single constrained baseline $(C=1)$ is used, and between $0 \%$ and $29 \%$ if two constrained baselines $(C=2)$ are used. Again we observe a larger improvement for weaker GNSS models.

Suboptimal versus Optimal Solution. As already mentioned in the introduction of this section, the suboptimal results are not included in the tables as these were exactly the same as optimal. Nevertheless we would like to report some observations on the difference between the suboptimal and optimal solution. In the suboptimal solution, the constrained baseline is not influenced by the unconstrained baseline; hence the result will be exactly the same as the uncoupled solution. We observed that the optimal solution and the suboptimal solution have almost the same performance for the success rate on individual baselines (i.e., Table 3): the maximum difference was $0.1 \%$ in the empirical success rates. Also for the overall success rate (i.e., results in Table 4), the optimal solution and the suboptimal solution have the same performance. This result is expected from the low correlation between the constrained and unconstrained baselines as discussed in Sections 4.3 and 4.4.

Triple versus Quadruple-Antenna Configuration. For the constrained baseline, the success rate for the uncoupled and optimal solution was found to be exactly the same (see Table 2). This table contains simulation results for the optimum solution on the triple-antenna configuration, but the same results are obtained for the quadruple-antenna configuration. The probability of obtaining the correct integer value increases as the precision of $\hat{a}$ improves. For the unconstrained baseline in the quadruple-antenna configuration ( $C=2$ case in Tables 3 and 4 ) we observe a higher success rate than for the same baseline in the triple antenna configuration ( $C=1$ case in Tables 3 and 4 ), which is as expected as the variance-covariance matrix is scaled with $1 / 2$ and 3/4, respectively, of the original $Q_{\hat{a}}$ of the uncoupled solution. Therefore we can say that the quadruple-antenna configuration is a stronger model than the triple-antenna configuration.

\section{Future Work}

The basic theory for a three- and four-antenna configuration, situated at two platforms with at most two antennas on a single platform, was developed in this contribution. As future work we will extend the method to be applicable with more antennas. The performance has to be evaluated with realistic noise values for code and carrier observation on moving vehicles including the effects of multipath. Furthermore, we 
will test the method using data collected in dedicated field experiments of challenging applications as vessel, air-, and spacecraft, where the unconstrained baseline between the platforms will vary rapidly over large distances $[5,34]$. In these field tests also the effect of remaining atmospheric delays, signal blocking, and multipath has to be investigated.

\section{Conclusions}

In this paper we explored methods for the combination of relative positioning and attitude determination for moving platforms, where each platform has multiantennas with known baseline lengths. The objective of this research was to develop a rigorous method that optimally makes use of all the information available (i.e., the integerness of the ambiguities, the relationship between the ambiguities on the different baselines, and the known baseline length of the constrained baselines) to determine the relative position and orientation of a multiantenna system with unconstrained and constrained baselines. In order to obtain more insight into the problem we investigated an uncoupled and two integrated strategies (coined the integer least squares or optimal, and vectorial bootstrapping or suboptimal approach) theoretically and experimentally. As was expected from the low correlation between the two baselines, the success rate of the integer least squares approach is similar to the vectorial bootstrapping approach. This was confirmed with simulated data for the single epoch, single frequency application. This is an important result as the suboptimal solution is more computational efficient and in general could be sufficient for the type of applications discussed in this paper. Furthermore we investigated triple- and quadruple-antenna configurations. The unconstrained baseline in the quadrupleantenna configuration provides a higher success rate than on the same unconstrained baseline in the triple-antenna configuration. This is as expected from the developed theory, as in the quadruple-antenna configuration two constrained baselines are placed at both sides of the unconstrained baseline, and in the triple-antenna configuration, only one constrained baseline is aiding the unconstrained baseline. The methods developed are rigorous and have the additional advantage that they improve ambiguity resolution on the unconstrained baseline(s) and the overall success rate of ambiguity resolution between a number of antennas.

\section{Acknowledgments}

Part of this work was done by Peter Buist at JAXAISAS, Japan, during a research visit with Professor Tatsuaki Hashimoto and the support of his group is acknowledged. The MicroNed-MISAT framework is kindly thanked for their support. Professor Teunissen is the recipient of an Australian Research Council Federation Fellowship (Project no. FF0883188). The research of Sandra Verhagen is supported by the Dutch Technology Foundation STW, Applied Science Division of NWO, and the Technology Program of the Ministry of Economic Affairs. This support is greatly acknowledged.

\section{References}

[1] T. M. Nguyen, J. W. Sinko, R. C. Galijan, S. R. I. Int, and M. Park, "Using differential carrier phase GPS to control automated vehicles," in Proceedings of the 40th Midwest Symposium on Circuits and Systems (MWSCAS '97), vol. 1, pp. 493-496, Sacramento, Calif, USA, August 1997.

[2] A. Kleusberg, "Kinematic relative positioning using GPS code and carrier beat phase observations," Marine Geodesy, vol. 10, no. 3-4, pp. 257-274, 1986.

[3] G. Campa, M. L. Fravolini, A. Ficola, M. R. Napolitano, B. Seanor, and M. G. Perhinschi, "Autonomous aerial refueling for UAVs using a combined GPS-machine vision guidance," in Proceedings of the AIAA Guidance, Navigation, and Control Conference and Exhibit, vol. 5, pp. 1-11, 2004.

[4] S. M. Khanafseh and B. Pervan, "Autonomous airborne refueling of unmanned air vehicles using the global positioning system," Journal of Aircraft, vol. 44, no. 5, pp. 1670-1682, 2007.

[5] P. J. Buist, "GNSS kinematic relative positioning for spacecraft: data analysis of a dynamic testbed," in Proceedings of the 26th International Symposium on Space Technology and Science (ISTS '08), Hamamatsu, Japan, June 2008.

[6] M. J. Vachon, R. J. Ray, K. R. Walsh, and K. Ennix, "F/A18 performance benefits measured during the autonomous formation flight project," Tech. Rep. NASA/TM-2003-210734, NASA Dryden Flight Research Center, Edwards, Calif, USA, 2003.

[7] J. L. Hansen, J. E. Murray, and N. V. Campos, "The NASA dryden AAR project: a flight test approach to an aerial refueling system," in Proceedings of the AIAA Atmospheric Flight Mechanics Conference and Exhibit, vol. 1, pp. 103-115, Providence, RI, USA, August 2004.

[8] B. Pervan, F. C. Chan, D. Gebre-Egziabher, S. Pullen, P. Enge, and G. Colby, "Performance analysis of carrier-phase DGPS navigation for shipboard landing of aircraft," Journal of Navigation, vol. 50, no. 3, pp. 181-191, 2003.

[9] C. E. Cohen, "Attitude determination," in Global Positioning System: Theory \& Applications, vol. 2, pp. 519-537, 1996.

[10] P. J. Buist, S. Kumagai, and K. Hama, "Flight experience of the integrated navigation unit; combining GPS attitude determination with star sensor measurements," in Proceedings of the 2nd ESA Workshop on Satellite Navigation User Equipment Technologies (NAVITEC '04), Noordwijk, The Netherlands, December 2004.

[11] G. Giorgi and P. J. Buist, "Single-epoch, single-frequency, standalone full attitude determination: experimental results," in Proceedings of the 4th ESA Workshop on Satellite Navigation User Equipment Technologies (NAVITEC '08), Noordwijk, The Netherlands, December 2008.

[12] G. Lachapelle, C. Liu, and G. Lu, "Quadruple single frequency receiver system for ambiguity resolution on the fly," in Proceedings of the 6th International Technical Meeting of the Satellite Division of the Institute of Navigation (ION GPS '93), 1993.

[13] J. Pinchin, C. Hide, D. Park, and X. Chen, "Precise kinematic positioning using single frequency GPS receivers and an integer ambiguity constraint," in Proceedings of the IEEE Position Location and Navigation Symposium (PLANS '08), pp. 600-605, 2008.

[14] http://www.javad.com/jgnss/products/receivers/triumph-4x .html.

[15] P. J. G. Teunissen and A. Kleusberg, GPS for Geodesy, Springer, Berlin, Germany, 2nd edition, 1998. 
[16] P. J. G. Teunissen, "The LAMBDA method for the GNSS compass," Artificial Satellites, vol. 41, no. 3, pp. 89-103, 2006.

[17] P. J. G. Teunissen, "Integer least squares theory for the GNSS compass," submitted to Journal of Geodesy.

[18] R. Hatch, "Instantaneous ambiguity resolution," in Proceedings of the International Symposium on Kinematic Systems for Geodesy, Surveying and Remote Sensing (KIS '90), Banff, Canada, December 1990.

[19] R. A. Brown, "Instantaneous GPS attitude determination," in Proceedings of the IEEE Position Location and Navigation Symposium (PLANS '92), pp. 113-120, April 1992.

[20] D. Knight, "A new method of instantaneous ambiguity resolution," in Proceedings of the 7th International Technical Meeting of the Satellite Division of the Institute of Navigation (ION GPS '94), vol. 1, pp. 707-716, Salt Lake City, Utah, USA, September 1994.

[21] C. Park, I. Kim, J. G. Lee, and G.-I. Jee, "Efficient ambiguity resolution using constraint equation," in Proceedings of the IEEE Position Location and Navigation Symposium (PLANS '96), pp. 277-284, Atlanta, Ga, USA, April 1996.

[22] E. Sutton, "Optimal search space identification for instantaneous integer cycle ambiguity resolution," in Proceedings of the 10th International Technical Meeting of the Satellite Division of the Institute of Navigation (ION GPS '97), vol. 1, pp. 313-322, Kansas City, Mo, USA, September 1997.

[23] D. Kim and R. B. Langley, "An optimized least-squares technique for improving ambiguity resolution and computational efficiency," in Proceedings of the 12th International Technical Meeting of the Satellite Division of the Institute of Navigation (ION GPS '99), Nashville, Tenn, USA, September 1999.

[24] M. S. Hodgart and S. Purivigraipong, "New approach to resolving instantaneous integer ambiguity resolution for spacecraft attitude determination using GPS signals," in Proceedings of the IEEE Position Location and Navigation Symposium (PLANS '00), pp. 132-139, San Diego, Calif, USA, March 2000.

[25] S. Draganov, B. Veytsman, and L. Haas, "Space applications algorithm and initial simulation results for the ITT low- power transceiver," in Proceedings of the 15th International Technical Meeting of the Satellite Division of the Institute of Navigation (ION GPS '02), Portland, Ore, USA, September 2002.

[26] P. Buist, S. Kumagai, T. Ito, K. Hama, and K. Mitani, "Development of the integrated navigation unit: combining a GPS receiver with star sensor measurements," in Proceedings of the 10th International Conference of Pacific Basin Societies (ISCOPS '03), vol. 117, 2003.

[27] P. Buist, "The baseline constrained LAMBDA method for single epoch, single frequency attitude determination applications," in Proceedings of the 20th International Technical Meeting of the Satellite Division of the Institute of Navigation (ION GNSS '07), vol. 3, pp. 2962-2973, Fort Worth, Tex, USA, September 2007.

[28] P. J. G. Teunissen, “The least-squares ambiguity decorrelation adjustment: a method for fast GPS integer ambiguity estimation," Journal of Geodesy, vol. 70, no. 1-2, pp. 65-82, 1995.

[29] P. J. G. Teunissen, "Least squares estimation of the integer GPS ambiguities," in Proceedings of the IAG General MeetingInvited Lecture, Section IV: Theory and Methodology, Beijing, China, August 1993.

[30] P. J. G. Teunissen, "A new method for fast carrier phase ambiguity estimation," in Proceedings of the IEEE Position Location and Navigation Symposium (PLANS '94), pp. 562573, Las Vegas, Nev, USA, April 1994.
[31] P. J. G. Teunissen, "An optimality property of the integer leastsquares estimator," Journal of Geodesy, vol. 73, no. 11, pp. 587593, 1999.

[32] C. Park and P. J. G. Teunissen, "A new carrier phase ambiguity estimation for GNSS attitude determination systems," in Proceedings of the International GPS/GNSS Symposium, Tokyo, Japan, 2003.

[33] G. Giorgi, P. J. G. Teunissen, and P. J. Buist, "A search and shrink approach for the baseline constrained LAMBDA: experimental results," in Proceedings of the International Symposium on GPS/GNSS, Tokyo, Japan, November 2008.

[34] P. J. Buist, S. Verhagen, T. Hashimoto, S. Sakai, and N. Bando, "GPS field experiment for the balloon-based operation vehicle," in Proceedings of the 18th Workshop on JAXA Astrodynamics and Flight Mechanics, Sagamihara, Japan, July 2008.

[35] S. Shimizu, P. J. Buist, N. Bando, S. Sakai, S. Sawai, and T. Hashimoto, "Design of multi-sensor attitude determination system for balloon-based operation vehicle," in Proceedings of the 27th ISTS International Symposium on Space Technology and Science, Tsukuba, Japan, July 2009.

[36] J. R. Magnus, Linear Structures, London School of Economics and Political Science, Charles Griffin, London, UK, 1988.

[37] P. J. G. Teunissen, "Success probability of integer GPS ambiguity rounding and bootstrapping," Journal of Geodesy, vol. 72, no. 10, pp. 606-612, 1998.

[38] S. Verhagen, "Visualization of GNSS-related design parameters: manual for the matlab user interface VISUAL," Internal document of MGP, Delft University of Technology, 2006, http://www.lr.tudelft.nl/mgp.

[39] P. J. G. Teunissen, "On the integer normal distribution of the GPS ambiguities," Artificial Satellites, vol. 33, no. 2, pp. 49-64, 1998. 

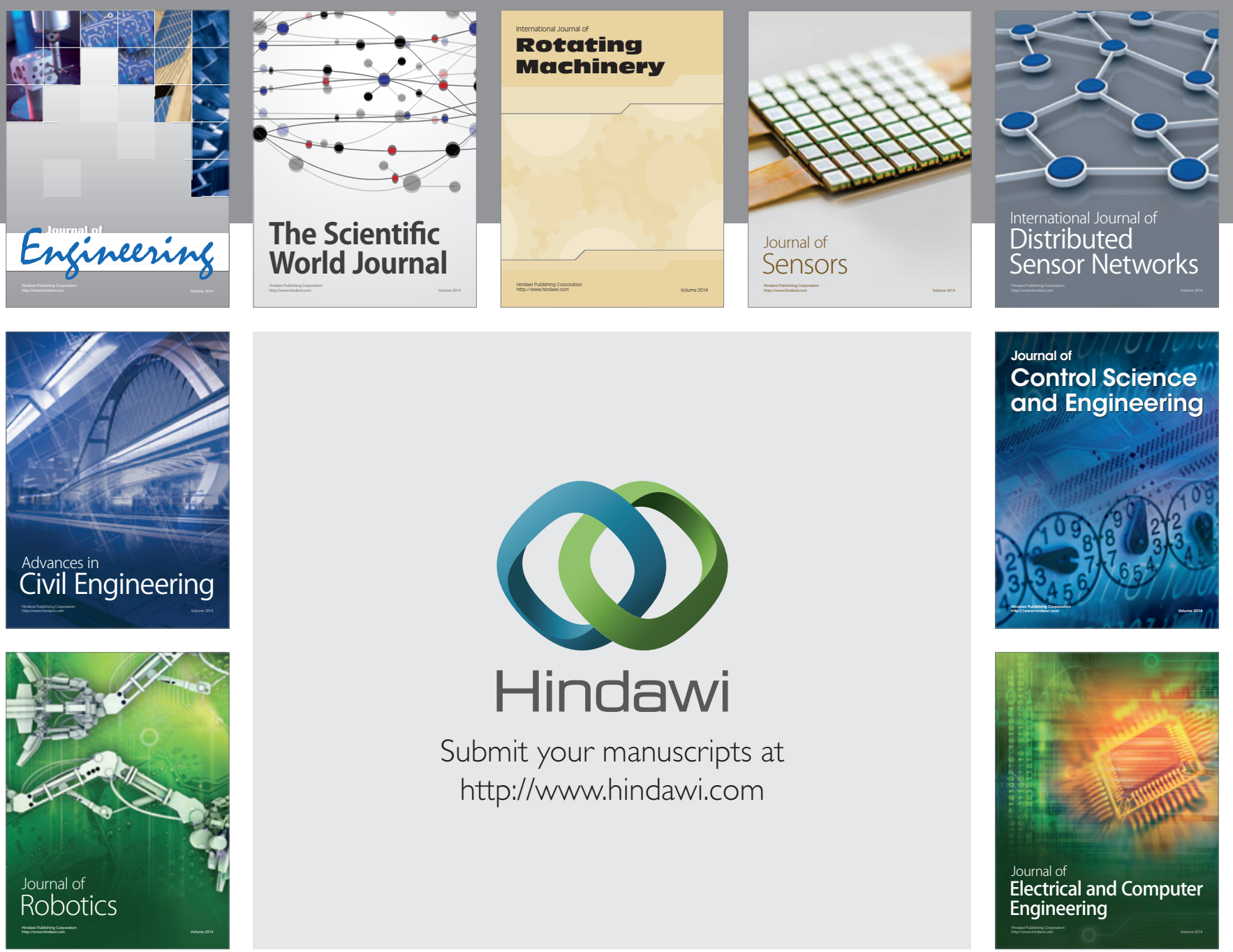

Submit your manuscripts at

http://www.hindawi.com
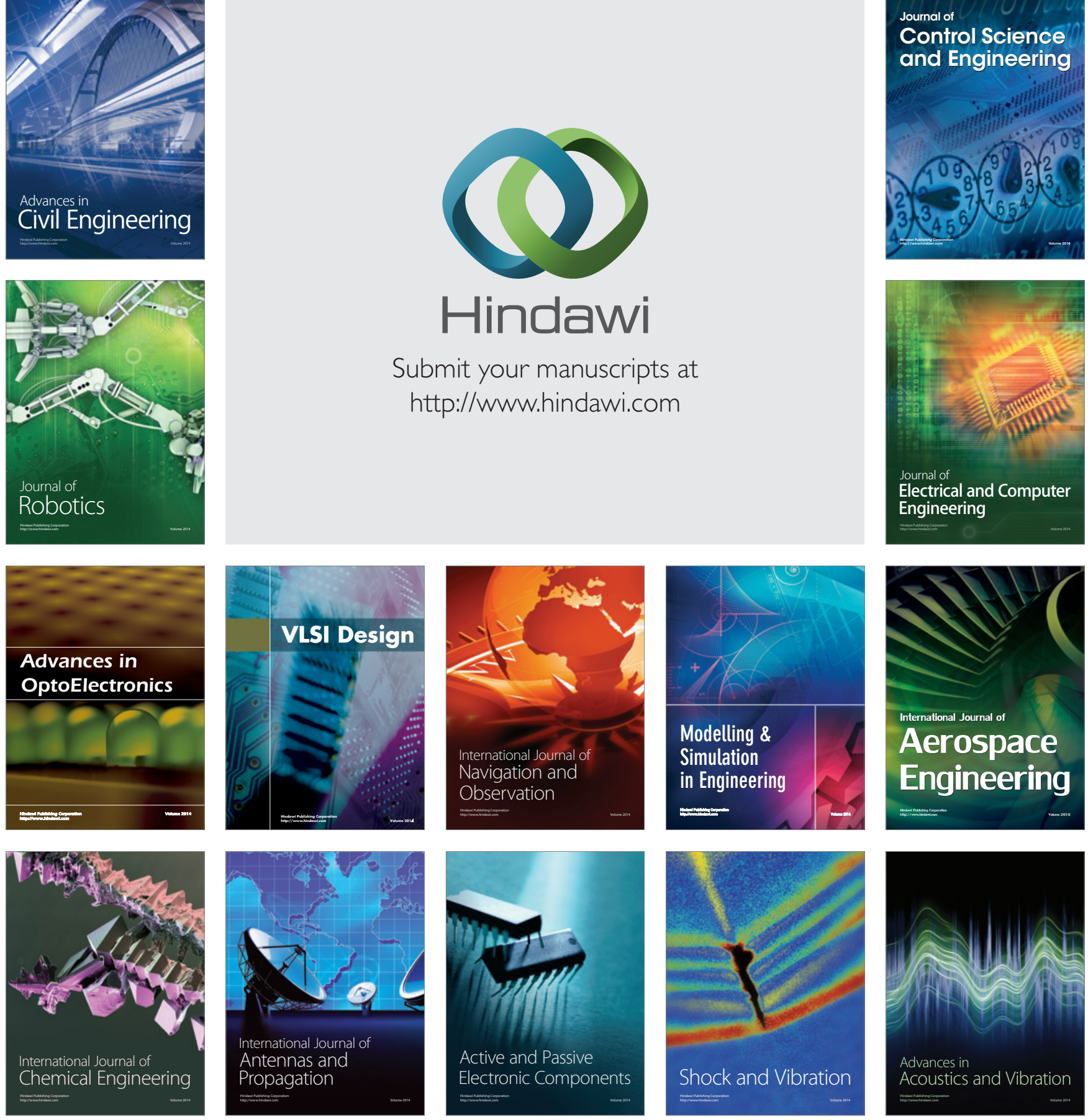